\title{
Reinventando a vida: proposta para uma abordagem sócio-antropológica do câncer de mama feminina
}

\author{
Reinventing life: a proposal for \\ a socio-anthropological approach \\ to breast cancer
}

Romeu Gomes 1

Márcia Marília Vargas Fróes Skaba 1,2

Roberto José da Silva Vieira 1

\footnotetext{
1 Instituto Fernandes Figueira, Fundação Oswaldo Cruz. Av. Rui Barbosa 716, 2 o andar, Rio de Janeiro, $R J$ 22250-020, Brasil. romeu@iff.fiocruz.br mhskaba@ccard.com.br rvieira@nitnet.com.br 2 Hospital do Câncer I, Instituto Nacional de Câncer. Praça Cruz Vermelha 23, Rio de Janeiro, $R J$ 202230-130, Brasil.
}

\begin{abstract}
This paper analyzes social representations of breast cancer and discusses the possibility of including symbolic aspects in the approach to this disease. The methodology is based on both a critical review of the theme (highlighting socio-anthropological issues) and the authors' experience in the field of oncology. The paper thus focuses on: (a) historical aspects of clinical and surgical approaches to breast cancer; (b) theoretical discussion of socially-constructed representations of cancer; and (c) incorporation of the symbolic dimension when dealing with women with breast cancer. The authors conclude that health care policy for women with breast cancer should consider both improvements in technical services and the symbolic dimension involved in living with this disease.
\end{abstract}

Key words Breast Neoplasms; Women's Health; Anthropology

Resumo O artigo objetiva analisar as representações sociais do câncer de mama e, com base nessa análise, discutir a possibilidade da inclusão de aspectos simbólicos na abordagem dessa doença. A metodologia, de um lado, fundamenta-se numa revisão crítica da temática, pontuando questões sócio-antropológicas e, de outro lado, baseia-se na experiência acumulada dos autores no campo da oncologia. Com base nesses princípios, o trabalho se configura a partir de (a) resgate de aspectos históricos das abordagens clínica e cirúrgica do câncer de mama; (b) discussão teórica da problemática à luz das representações socialmente construídas em torno do câncer; (c) considerações sobre a incorporação da dimensão simbólica na abordagem de mulheres com neoplasias de mama. Os autores concluem que as políticas de atenção às mulheres com neoplasias mamárias devem formular princípios para que se possa lidar, ao mesmo tempo, com o aperfeiçoamento das intervenções técnicas e da dimensão simbólica construída na trajetória de ser portador dessa doença.

Palavras-chave Neoplasias Mamárias; Saúde da Mulher; Antropologia 


\section{Considerações iniciais}

No Brasil, o câncer se apresenta como uma expressiva causa de mortalidade. Fonseca (1995) observa que, em 1950, as mortes dividiam-se em 14,2\% relacionadas a doenças do coração; $5,7 \%$ a cânceres e $35,9 \%$ a doenças infecciosas e parasitárias. No final da década de 80 , essas proporções foram alteradas, respectivamente, para $32,4 \%, 12,6 \%$ e 6,0\%. Em 1997, as neoplasias femininas continuavam sendo a terceira causa de morte entre as mulheres, para todo o Brasil, segundo dados do Sistema de Informação sobre Mortalidade (DATASUS, 1997).

Especificamente em relação ao câncer de mama, nos anos 80, a incidência foi alta nos Estados Unidos e no Norte da Europa, com 104 casos em 100.000 mulheres e 73,5 casos em 100.000 mulheres, respectivamente (Tessaro, 1999). Outro fato importante é que, até 1980, a maioria dos aumentos nas taxas de incidência era verificada em mulheres com mais de cinqüenta anos, mas, no período de 1980 a 1987, as taxas de incidência também aumentaram em mulheres mais jovens (MS, 2000).

Ainda em referência ao câncer de mama, as taxas de incidência são mais altas na América do Norte e no Norte da Europa, intermediárias no Sul da Europa e América do Sul e mais baixas na Ásia e na África. Entretanto, tem havido aumento significativo em diversos países da Ásia. No Japão, a taxa de incidência ajustada para idade foi mais que o dobro no período de 1983 a 1987, comparando-se com o período de 1969 a 1972 (Vieira, 1999).

Segundo estimativas do Instituto Nacional de Câncer (MS, 2000), para o ano de 2000, no Brasil, estima-se que o câncer de mama feminina seja o segundo tipo de câncer mais incidente, com 28.340 casos. Esse tipo de neoplasia maligna será, ainda, o de maior taxa de incidência entre a população feminina brasileira, com 33,58 em 100.000 mulheres.

Atualmente, no Brasil, apenas 3,4\% dos casos de câncer de mama são detectados na fase inicial, enquanto $60 \%$ são diagnosticados em casos avançados, quando a doença já se tornou incurável (MS, 2000).

Estudos epidemiológicos indicam que fatores ambientais são responsáveis por, pelo menos, $80 \%$ da incidência do câncer de mama. Fatores genéticos representam de $5 \%$ a $7 \%$ de sua etiologia, porém, quando se apresenta antes dos 35 anos, esta freqüência chega a 25\% (MS, 2000).

Diante de tão preocupante quadro, em torno do câncer, em geral, e em torno do câncer de mama feminina, em específico, há todo um investimento simbólico que é socialmente cons- truído. Por isso, é de fundamental importância que a saúde pública contemple também, em sua agenda, a dimensão sócio-antropológica acerca dessa problemática.

A partir dessas considerações, objetivamos analisar as representações sociais do câncer de mama e, com base nessa análise, discutir a possibilidade da inclusão de aspectos simbólicos na abordagem dessa doença. No bojo desta discussão, esperamos contribuir para um olhar mais ampliado das políticas oncológicas voltadas às mulheres com neoplasias mamárias. Em outras palavras, buscamos contribuir para o deslocamento da exclusividade do foco terapêutico dessas políticas, predominantemente baseado no modelo biomédico, para a incorporação de práticas preventivas que contemplem significados do sujeito demarcados por suas relações sociais.

Nosso referencial teórico-metodológico baseia-se no conceito das representações sociais, aqui entendidas como "um tipo de saber, socialmente negociado, contido no senso comume na dimensão cotidiana, que permite ao indivíduo uma visão de mundo e o orienta nos projetos de ação e nas estratégias que desenvolve em seu meio social" (Queiroz, 2000:27).

Para melhor compreendermos como as representações sociais são construídas e como se refletem nas ações dos sujeitos e dos grupos sociais, remetemo-nos ao conceito de habitus de Pierre Bourdieu, entendido como "sistemas de disposições socialmente constituídas que, enquanto estruturas estruturadas e estruturantes, constituem o princípio gerador e unificador do conjunto das práticas e das ideologias características de um grupo de agentes" (Bourdieu, 1992:191). Assim, esse conceito se refere a um conhecimento adquirido, um haver, um capital, indicando "uma disposição incorporada, quase postural” (Bourdieu, 1998:61), revelada na construção histórica da produção das práticas. Ao contrário da palavra "hábito", que se associa a algo cristalizado, a expressão habitus envolve uma capacidade criadora, ativa e inventiva.

Respaldados em Bourdieu, demarcamos a diferença entre "hábito" e habitus, observando que faz-se necessário abandonar posicionamentos que apontem para uma concepção de prática como uma reação determinada apenas por condições antecedentes e redutível aos aspectos mecânicos.

Para esse autor, o conhecimento do mundo social tem que tomar em conta o conhecimento prático que lhe preexiste, embora esse conhecimento seja, num primeiro momento, permeado por representações parciais. Conside- 
rando os diferentes contextos históricos e cenários culturais, ele considera que o habitus expressa uma herança naturalizada que permeia as relações sociais. Entretanto, ainda segundo o autor em questão, o agente social não é um mero reprodutor dessa herança, nem é um mero executor de algo que se encontra objetivamente programado e que lhe é exterior.

Herzlich (1991) observa que, embora Bourdieu confira um peso maior ao passado, dimensão pouco explorada pelos estudos das representações sociais, esta teoria não se afasta totalmente do conceito bourdiano de habitus.

Nesse sentido, entendemos as representações sociais ou habitus como um conceito dinâmico, sintetizador de estruturas cristalizadas, mas com possibilidades de reivenção, que opera na mediação entre estruturas objetivas e a reconstrução da ordem simbólica, subjetiva. Sendo assim, poderíamos inferir que essa matriz teórica aponta caminhos férteis para compreendermos como as representações sociais sobre o câncer foram historicamente construídas e quais as possibilidades de transformações nesse campo (Skaba, 2001).

Com base nessa perspectiva teórica, podemos, ainda, compreender as representações do câncer de mama como um produto das relações entre significados, socialmente construídos, em torno do câncer em geral, do corpo feminino em específico e da experiência de ser portador dessa patologia.

Em termos metodológicos, delineamos o presente estudo como uma revisão crítica acerca da temática que nos propomos discutir. Para isso, procuramos pontuar aspectos teóricos para que possam dar suporte ao levantamento de questões sócio-antropológicas que emergem dessa discussão. Por outro lado, nos baseamos na experiência acumulada dos autores no campo da oncologia.

Com base nesses princípios, o presente trabalho se configura a partir de (a) resgate de aspectos históricos das abordagens clínica e cirúrgica do câncer de mama; (b) discussão teórica da problemática à luz das representações socialmente construídas em torno do câncer; (c) considerações sobre a incorporação da dimensão simbólica na abordagem de mulheres com neoplasias de mama.

\section{Um pouco da história do tratamento do câncer de mama}

A fim de situarmos o avanço do tratamento do câncer de mama, com base em Dias (1994), Donegan (1995) e Veronesi et al. (1992), resga- tamos a seguir aspectos históricos sobre essa neoplasia.

A história sobre o tratamento do câncer de mama é antiga. Já no Egito, aproximadamente no ano 2500 a.C., em um papiro, que descrevia e prescrevia casos de inúmeras doenças, encontrava-se o seguinte registro: "Uma mama com tumor protuberante e fria ao toque representa uma doença para a qual não há tratamento" (Dias, 1994:3). Também Hipócrates, que dedicou parte de sua obra ao câncer de mama e o considerava uma doença incurável, em 460 a.C., não recomendava qualquer tipo de tratamento.

A primeira cirurgia de mama foi realizada no século I d.C. pelo médico grego chamado Leonidas, e no mesmo século o enciclopedista romano Aurelius Cornelius Celsus afirmava que a cirurgia era inútil quando a doença estava ulcerada. Teve-se a primeira esperança quanto ao tratamento do câncer de mama no século II, quando Galeno, considerado o maior médico grego depois de Hipócrates, afirmava ser possível curar o câncer de mama pela cirurgia, desde que o tumor fosse superficial e todas as suas raízes extirpadas.

No século XVIII, novas técnicas cirúrgicas são descritas trazendo contribuições importantes como ligadura de vasos e a criação de instrumental específico para a cirurgia de mama, com artigos publicados por ilustres nomes da medicina tais como: Versalenis, Ambroise, Parré, Michel Servetus e Wilhelm Fabry.

Ainda nesse século, os cirurgiões abusaram das indicações de mastectomias e as realizaram em grande número, caindo em descrédito em virtude dos péssimos resultados alcançados.

No século X, Avicena, conhecido como Príncipe dos Médicos um dos mais importantes nomes da história da Medicina, acreditava que a cirurgia era válida apenas para casos superficiais.

No Renascimento, Lorenj Heister defendia o uso de uma guilhotina para tornar a mastectomia um procedimento mais rápido e menos doloroso. A primeira abordagem médico-paciente foi feita por ele, que dizia: "muitas $m u$ lheres podem tolerar a operação com a maior coragem e sem gemer absolutamente. Outras, entretanto, fazem um escândalo tal que pode desencorajar o mais destemido dos cirurgiões $e$ dificultar a operação. Para realizá-la, o cirurgião, portanto, deve ser persistente e não permitir-se desconcentrar-se com o choro da paciente" (Heister, apud Dias, 1994:3).

Com a descoberta da anti-sepsia por Joseph Lister e da anestesia por Willian Moston (Estados Unidos), em 1846, a medicina teve um gran- 
de avanço e foram amenizados os grandes terrores da cirurgia que eram a dor e a infecção. A anatomia patológica teve também seu grande avanço quando, pela primeira vez, um tumor de mama foi visto por um microscópio e os primeiros estudos foram publicados, trazendo o aperfeiçoamento de técnicas cirúrgicas baseados em conhecimentos anatômicos mais precisos.

O primeiro a irradiar uma paciente com câncer de mama foi o estudante de medicina Emile Grubbe, em Boston, no ano de 1895, mas só no século seguinte, na década de 20 , a radioterapia é introduzida no período pós-operatório da mastectomia radical. Em 1898, Paul Erlich, considerado o pai da quimioterapia, isola o primeiro agente alquilante.

Em 1889, o cirurgião americano William Stewart Halsted (Baltimore) descreve sua técnica de mastectomia radical que permanece como padrão de tratamento de câncer de mama por mais de setenta anos.

No ano de 1895, foi feita a primeira biópsia de congelação pelo patologista americano Willian Welch, e Vicenz Czerny realiza a primeira reconstrução mamária, transportando um grande lipoma para a região da mama amputada. No ano seguinte, Tansini, da Universidade de Pádua (Itália), descreve a primeira tentativa de reconstrução da parede tóracica anterior após a mastectomia.

No início do século XX, ocorreram várias modificações das técnicas cirúrgicas já descritas anteriormente, iniciando-se a colocação da prótese de silicone.

Com base em estudos desenvolvidos no Guy's Hospital (Londres), chegou-se à conclusão de que a cirurgia conservadora alcançava os mesmos objetivos das cirurgias radicais, e estudos estatísticos comprovaram que a sobrevivência era praticamente a mesma. Esses trabalhos foram aprimorados pelo professor Veronesi, do Instituto de Milão (Itália).

A técnica de quadrantectomia teve início com grandes ressecções de quadrantes mamários, passando, em seguida, para intervenções mais moderadas aprimorando a técnica reparadora, quando melhores resultados estéticos foram alcançados. Em 1981, foi publicado o primeiro trabalho em que se provava a igualdade entre a mastectomia radical e a cirurgia conservadora para determinados tipos de tumores. Segundo Donegan (1995), Bernie Fisher, em um estudo multicêntrico, realizado no Canadá e nos Estados Unidos da América, confirma a veracidade das publicações em Milão. Este estudo propunha quatro níveis de informações, a saber: estudar a sobrevivência global de dois grupos de pacientes; pesquisar o índice de recidiva regional à distância; verificar a incidência da recidiva local e, fundamentalmente, avaliar o resultado cosmético das pacientes tratadas com quadrantectomia.

Atualmente, é consenso que a cirurgia conservadora terá sempre sucesso se tiver uma indicação clínica precisa. O tumor deve ser único; seu tamanho não deve passar de $3 \mathrm{~cm}$ de diâmetro e a ressecção do mesmo deverá ter margens menores de $2 \mathrm{~cm}$ livres. Os fatores prognósticos do tumor e axila decidem o tratamento adjuvante (quimioterapia ou hormonioterapia).

A mastectomia não foi abandonada; em casos como tumores multicêntricos, tumores centrais (de localização retroareolares) e com desproporção entre o tamanho do tumor e o tamanho da mama, essa técnica é ainda indicada.

Quando feita indicação de tratamento cirúrgico conservador da mama, deve-se procurar observar o baixo índice de recidiva local (até $10 \%$ ), uma boa margem de segurança da ressecção tumoral e principalmente um bom resultado estético.

Durante setenta anos, a Escola de Halsted, baseada no princípio científico de que a mutilação era considerada a cura do câncer, e por este motivo a extirpação da mama e da região adjacente assegurava à paciente uma sobrevida maior, legou à humanidade uma terrível visão do tratamento do câncer de mama.

Ao tratar uma paciente com tamanha agressividade, deixando sempre seqüelas como o edema do braço, que a diferenciava da população em geral, restringindo sua vida social, fez com que as demais - não portadoras desta doença - sequer falassem sobre a mesma.

Com o avanço do diagnóstico precoce do câncer de mama através de tecnologia avançada de imagem - como a mamografia, a ultrasonografia, a ressonância magnética, a punção biópsia, a estereotaxia para a marcação de nódulos ou áreas de microcalcificações de diminutos tamanhos - houve a possibilidade do diagnóstico inicial e ainda restrito da doença sem ter se propagado pelo corpo, deixando de ser uma doença sistêmica para ser uma doença localizada. Sendo assim, a necessidade da mastectomia deixou de ser um imperativo para o tratamento do câncer de mama, podendo ser retirada apenas a área afetada, e proporcionando à mulher a preservação de um órgão de tão grande importância para a sua feminilidade.

Apesar de toda essa evolução, observamos que a suspeita do câncer de mama e a indicação de todos esses procedimentos ainda causam medos e crises em muitas portadoras dessa doença. No imaginário social, a mama costuma ser associada a atos prazerosos - como 
amamentar, seduzir e acariciar -, não combinando com a idéia de ser objeto de uma intervenção dolorosa, ainda que necessária.

A máquina foi criada, os recursos estão presentes, mas a relação máquina-mulher ainda não teve seu ajuste final. A mamografia, que é um exame totalmente controlado por um computador, fazendo com que um aparelho comprima a mama de tal forma que sejam visualizadas todas as suas estruturas, é feita após a indicação médica que tenta convencer a mulher de que esta é a única forma que temos para detectar alguma modificação inicial da imagem. Mesmo assim, é comum na população feminina considerar esse exame como algo altamente desagradável.

Falta, assim, paralelamente ao desenvolvimento de tecnologias de última geração que busquem obter o máximo de eficácia terapêutica com a ausência ou a redução ao mínimo de desconforto, avançar no campo de um conhecimento que contemple a dimensão simbólica que é construída em torno desses procedimentos.

\section{As representações sociais do câncer}

Sontag (1984:7) inicia seu trabalho sublinhando que "a doença é o lado sombrio da vida, uma espécie de cidadania mais onerosa. Todas as pessoas vivas têm dupla cidadania, uma no reino da saúde e outra no reino da doença". O câncer tem um lugar privilegiado neste "lado sombrio da vida”, sendo freqüentemente interpretado como uma doença insidiosa e arrogante que transforma a vida das pessoas acometidas por essa enfermidade (Skaba, 2000).

Historicamente o câncer vem sendo associado a experiências malditas e servindo como metáforas para diversas ordens de infortúnios físicos, mentais e sociais. Freqüentemente somos lembrados dos diferentes tipos de "cânceres” sociais. A violência, a desintegração familiar, o uso de drogas e a corrupção têm sido referidos como perturbadores da ordem e, conseqüentemente, denominados câncer.

Sontag (1984) observa, ainda, que o câncer tem sido visto como uma doença cruel, intratável e misteriosa. Por ser algo que ataca, invade, o corpo, o seu tratamento tem sido pensado como um contra-ataque, fazendo com que muitas vezes seja visto como algo pior do que a própria doença. Nesse tratamento, costuma-se usar metáforas tiradas da linguagem militar. Assim, a radioterapia é o bombardeamento com raios tóxicos e a quimioterapia objetiva matar as células cancerosas.
Nessa "guerra", segundo a autora, quase todo dano causado ao corpo é justificado se a meta for salvar vidas. Essa abordagem, em parte, pode ser creditada ao fato de o modelo médico, em geral, conceber as doenças como entidades universais biológicas ou psicofisiológicas, resultantes de disfunções somáticas. Dentro desse raciocínio, a medicina clínica visa, principalmente, interpretar sintomas, relacionando-os a fontes funcionais e estruturais do corpo, e a subjacentes entidades doentes, para chegar a um diagnóstico. Em seguida, a tarefa da clínica médica é o tratamento racional visando intervenção nos mecanismos doentes (Good, 1994).

A despeito das crescentes possibilidades terapêuticas para o câncer, subjaz ao seu significado a idéia de algo que cresce e destrói, sendo associado em muitas culturas à punição e ao castigo. Essas crenças podem trazer conseqüências desastrosas, tanto do ponto de vista emocional, como da ação prática e concreta para o enfrentamento da possibilidade de adoecer por câncer.

Françoso (1993:21), com base em Kato, analisa as repercussões do significado emprestado ao câncer e sublinha que "o adulto, por ter uma representação da doença como fatal, antecipa a possibilidade de perda por morte desenvolvendo um processo denominado luto antecipatório - processo pontuado pela incerteza e por desinvestimentos de grau variado". Nessa perspectiva, a sua cura é concebida como um milagre e não como fenômeno possível de ocorrer em virtude dos avanços da área médica. Sendo assim, a cura, enquanto realidade, ainda não foi incorporada ao repertório cultural.

Em estudo realizado com mulheres mastectomizadas, Silva \& Mamede (1998:47) percebem que "o medo está em todas as fases percorridas pela mulher no processo de adoecer. O medo do diagnóstico câncer torna-se ameaçador, originando, assim, reações emocionais, que provocarão mudanças no âmbito biológico, mental e social".

Com base em Sontag, Schulze (1993) observa que, na discussão acerca do câncer, deve-se levar em conta a questão do estigma social, que faz com que os cancerosos sejam vistos como pessoas que vão morrer por conta de sua doença.

Em face desse imaginário social, a abordagem do câncer envolve sentimentos que são difíceis de ser administrados, tanto por parte dos profissionais, como por parte das pessoas em geral. Medo da dor, resignação diante da percepção de que sua doença terá um desfecho fatal e a negação de que a própria doença existe 
são alguns dos aspectos emocionais que envolvem toda a trajetória de ser portador de câncer.

Junto à dimensão psicológica, há questões sociais que devem ser apontadas no debate em questão. Silva \& Mamede (1998) observam que, diante do enfrentamento da situação, as entrevistadas expressaram um movimento de organizar os seus relacionamentos sociais. Nesse movimento, pode constituir-se uma rede de pessoas, envolvendo família, amigos, profissionais de saúde e grupos de apoio: "os componentes dessa rede de suporte poderão facilitar ou não o estabelecimento de laços afetivos e sociais necessários e imprescindiveis ao enfrentamento das dificuldades" (Silva \& Mamede, 1998:105).

Assim, por conta de toda a problemática que envolve o tema, a discussão não pode ser restrita a aspectos da clínica médica. Nela deve ser contemplada a necessidade de se considerarem os desdobramentos sociais que seguem à descoberta do câncer, demandando ações de saúde pública voltadas para essa doença.

O câncer de mama feminina está entre as doenças que mais afetam a mulher, fazendo com que suas portadoras vivenciem um processo de fragilização. Para que os profissionais de saúde possam lidar com essa problemática, é preciso que haja, além do entendimento da dimensão psicológica, uma compreensão sócio-antropológica do contexto e da situação social em que se encontra o sujeito da doença, uma vez que é através das interações sociais que "indivíduos desenvolvem determinadas experiências, estabelecem campos de significação e adquirem um senso de sua própria identidade" (Hita, 1998:179).

Quando tratamos do câncer de mama feminina, acrescentamos aos fatores relacionados ao câncer em geral os aspectos relacionados à função social do corpo da mulher. Nesse sentido, no universo simbólico, a mama é um elemento fundamental capaz de concentrar papéis da identidade feminina, como a sexualidade - incluída aí a sensualidade -, sendo os seios objeto de prazer e desejo, e a feminilidade diante da possibilidade exclusivamente concedida à mulher do ato da amamentação.

A possibilidade de desenvolver uma doença na mama compromete toda essa construção fundamental da existência feminina. Compreender esses significados colabora no entendimento da totalidade dos aspectos que compõem o adoecimento por câncer, incorporando ao tratamento do tumor o sujeito fragilizado em suas funções de mulher e mãe.

Com base em nossa experiência na abordagem a mulheres portadoras de câncer de ma- ma, podemos refletir sobre as repercussões que as representações sociais sobre essa patologia trazem para as mulheres, no sentido de se criarem condições, objetivas e subjetivas, para que haja mobilização em busca de um caminho que favoreça o tratamento e o enfrentamento do câncer.

Freqüentemente, a mulher, ao se perceber como possível portadora de uma patologia representada socialmente como aliada à dor ao sofrimento e com desfecho fatal, sofre reflexos dessas construções historicamente determinadas, que podem muitas vezes levá-la à negação desse processo, traduzindo-se em um elemento dificultador para a detecção precoce do câncer e, conseqüentemente, para a obtenção de melhores prognósticos.

A compreensão dessa teia de significados pode sinalizar para uma promoção em saúde que invista na desconstrução dessas representações, passando pela busca de possibilidades de se lidar com a doença como algo passível de controle, assegurando melhor qualidade de vida a essas pacientes.

\section{Considerações finais: a incorporação dos aspectos simbólicos na abordagem de mulheres com câncer de mama}

Com base em nossa reflexão, propomos uma reinvenção das representações sociais sobre o câncer de mama. Compartilhamos da perspectiva de análise sustentada por Pierre Bourdieu. Operamos, sobretudo, com o conceito de habitus, que nos orienta a pensar que as práticas sociais e suas representações têm a marca da sua historicidade, mas que a criatividade humana tem possibilidades de reconstruir paradigmas.

Nessa perspectiva, reafirmamos o já conhecido problema da saúde pública para proporcionar os meios objetivos para prevenção, diagnóstico precoce e a oferta de serviços capazes de efetivar o controle do câncer de mama, traduzido pelas dificuldades de acesso, sobretudo nas camadas economicamente menos favorecidas. Tais dificuldades se expressam em intermináveis filas, problemas para marcação de consultas, longa espera para atendimento, problemas nas interações entre profissionais de saúde e usuários e problemas relacionados a resultados de exame.

Por meio dessa discussão, pretendemos trazer o olhar das representações sociais, numa perspectiva complementar, para que o senso comum sobre a patologia possa ser trabalhado, no campo da prevenção e da promoção da saú- 
de, a fim de apontar possibilidades de melhor enfrentar o câncer de mama.

Sendo assim, sublinhamos o papel dos profissionais de saúde e dos formuladores de políticas preventivas na desconstrução, dentre outros aspectos, do inseparável binômio câncer de mama e mutilação. Apostamos que a prevenção e a detecção precoce só serão incorporadas às práticas de cuidados com o corpo feminino se as mulheres vislumbrarem possibilidades efetivas de melhores prognósticos.

Dialeticamente, propomos que as construções simbólicas sobre o câncer de mama incorporem também as construções efetivas de diagnóstico e tratamento disponíveis atualmente. O controle do câncer, como uma realidade possível para certas condições, necessita ser incorporado ao repertório cultural da vida em sociedade.

Se o apelo à informação por intermédio da divulgação do desenvolvimento científico, conquistado pela ciência oncológica contemporânea, pode ser um caminho para a reconstrução das representações sociais sobre o câncer de mama, não podemos desconsiderar a incorporação dos aspectos simbólicos para a compreensão desta problemática.

Concomitante aos avanços tecnológicos conquistados para o controle do câncer de mama, faz-se necessário igual avanço no campo do conhecimento que busque trabalhar a rela- ção dos sujeitos da doença com tais resultados, de forma que possamos avaliar as mudanças ocorridas e as que serão necessárias no âmbito tanto das máquinas, como das atitudes que emergem dessa relação.

Compreender o avanço tecnológico sobre determinado campo impõe uma nova definição para o conceito de tecnologia, conforme sublinhou Gonçalves (1994). Este autor conceitua tecnologia "enquanto conjunto de saberes e instrumentos que expressa, nos processos de produção de serviços, a rede de relações sociais em que seus agentes articulam sua prática em uma totalidade social" (Gonçalves, 1994:32). Assim, é necessário incorporar ao desenvolvimento tecnológico não apenas os instrumentos materiais disponíveis, mas também a construção dos saberes em sentido ampliado, que possibilite, dentre outros aspectos, lidar com a dimensão simbólica que surge em torno e em decorrência da materialidade da tecnologia.

Com base nessa discussão, em face da compreensão do câncer de mama como uma patologia permeada por uma complexidade de fenômenos, concluímos que as políticas de atenção às mulheres com neoplasias mamárias devem formular princípios para que se possa lidar, ao mesmo tempo, com o aperfeiçoamento das intervenções técnicas e a dimensão simbólica construída na trajetória de ser portador dessa doença.

\section{Referências}

BOURDIEU, P., 1992. A Economia das Trocas Simbólicas. São Paulo: Editora Perspectiva.

BOURDIEU, P., 1998. O Poder Simbólico. Rio de Janeiro: Editora Bertrand Russel.

DATASUS (Departamento de Informática do SUS), 1997. Sistema de Informação sobre Mortalidade 1979 -1997: Dados de Declaração de Óbito. CDROM. Brasília: Ministério da Saúde.

DIAS, E. N., 1994. A mastologia através da história. In: Mastologia Atual (E. N. Dias, M. Caleffi, H. M. S. Silva \& A. S. S. Figueira Filho, org.), pp. 3-6, Rio de Janeiro: Editora Revinter.

DONEGAN, W. L., 1995. Introduction to the history of breast cancer. In: Cancer of the Breast (W. L. Donegan \& J. S. Spratt, ed.), pp. 1-15, Philadelphia: W. B. Saunders Company.

FONSECA, L. A. M., 1995. A evolução das doenças neoplásicas. In: Velhos e Novos Males da Saúde no Brasil (C. A. Monteiro, org.), pp. 268-278, São Paulo: Editora Hucitec. 
FRANCCOSO, L. P. C., 1993. Enfermagem: Imagens $e$ Significados do Câncer Infantil. Dissertação de Mestrado, Ribeirão Preto: Escola de Enfermagem de Ribeirão Preto, Universidade de São Paulo.

GONÇALVES, R. B. M., 1994. Tecnologia e Organização Social das Práticas de Saúde: Características Tecnológicas do Processo de Trabalho na Rede Estadual de Centros de Saúde de São Paulo. São Paulo: Editora Hucitec/Rio de Janeiro: ABRASCO.

GOOD, B. J., 1994. Medicine, Rationality and Experience: An Antropological Perspective. Cambridge: Cambridge University Press.

HERLIZCH, C., 1991. A problemática da representação social e sua utilidade no campo da doença. Physis, 1:23-36.

HITA, M. G., 1998. Identidade feminina e nervoso: Crises e trajetórias. In: Antropologia da Saúde: Traçando Identidade e Explorando Fronteiras (P. C. Alves \& M. C. Rabello, org.), pp. 179-213, Rio de Janeiro: Editora Fiocruz/Editora Relume Dumará.

MS (Ministério da Saúde), 2000. Estimativas da Incidência de Mortalidade por Câncer no Brasil 2000. Rio de Janeiro: Instituto Nacional de Câncer.

QUEIROZ, M. S., 2000. Representações sociais: Uma perspectiva multidisciplinar em pesquisa qualitativa. In: Doenças Endêmicas: Abordagens Sociais, Culturais e Comportamentais (R. B. Barata \& R. Briceño-León, org.), pp. 27-45, Rio de Janeiro: Editora Fiocruz.

SCHULZE, C. M. N., 1993. As representações sociais de pacientes portadores de câncer. In: O Conhecimento no Cotidiano: As Representações Sociais na Perspectiva da Psicologia Social (M. J. Spink, org.), pp. 266-279, São Paulo: Editora Brasiliense.
SILVA, R. M. \& MAMEDE, M. V., 1998. Conviver com a Mastectomia. Fortaleza: Editora da Universidade Federal do Ceará.

SKABA, M. M. V. F., 2000. Em Busca do Diagnóstico: Representações Sociais, Prevenção e Detecção Precoce do Câncer de Mama no Brasil. Anteprojeto de Tese de Doutorado, Rio de Janeiro: Pós-Graduação em Saúde da Criança e da Mulher, Instituto Fernandes Figueira, Fundação Oswaldo Cruz. (mimeo.)

SKABA, M. M. V. F., 2001. As Representações Sociais e sua Utilização como Instrumental para a Compreensão do Processo de Adoecimento: O Caso da Oncologia. Rio de Janeiro: Pós-Graduação em Saúde da Criança e da Mulher, Instituto Fernandes Figueira, Fundação Oswaldo Cruz. (mimeo.)

SONTAG, S. A., 1984. Doença como Metáfora. Rio de Janeiro: Editora Graal.

TESSARO, S., 1999. Epidemiologia do câncer de mama. In: Câncer de Mama (D. L. Basegio, org.), pp. 1-11, Rio de Janeiro: Editora Revinter.

VERONESI, U.; LUINI, A. \& ANDREOLI, C., 1992. A Conservação da Mama: Indicações e Técnicas da Quadrantectomia, Dissecção e Radioterapia no Câncer de Mama. São Paulo: Editora Ícone.

VIEIRA, R., 1999. Agregação Familial de Câncer de Mama e Ovário: Estudo Descritivo em Amostras de Famílias no Brasil. Rio de Janeiro: Editora Revinter.

Recebido em 2 de janeiro de 2001

Versão final reapresentada em 17 de abril de 2001 Aprovado em 27 de julho de 2001 